\title{
Molecularly imprinted polypyrrole sensors for the detection of pyrene in aqueous solutions
}

\author{
Olwethu W. Ngwanya ${ }^{1} \cdot$ Meryck Ward ${ }^{1} \cdot$ Priscilla G. L. Baker ${ }^{1}$ \\ Accepted: 9 December 2020 / Published online: 13 January 2021 \\ (C) The Author(s), under exclusive licence to Springer Science+Business Media, LLC part of Springer Nature 2021
}

\begin{abstract}
Recently, electrochemical sensors have emerged as tools for polyaromatic hydrocarbons (PAH) detection that are cost-effective, easy to produce and use, highly selective and sensitive, and with good reproducibility. Polypyrrole may be easily produced from polymerization of pyrrole, by chemical as well as electrochemical methods, to produce dimensionally stable semi-conductive polymer materials, under mild synthesis conditions. In this study, polypyrrole was used as the stable molecular framework within which to create an imprint of the desired polyaromatic hydrocarbon, in situ, at glassy carbon electrodes. The molecularly imprinted polymer (MIP) sensors were washed to remove the imprint and subsequently characterized by atomic force microscopy (AFM), scanning electron microscopy (SEM), and cyclic voltammetry (CV). The MIP sensors were then applied to the detection of pyrene and non-imprinted polymers (NIP) sensors were also evaluated for comparison with the MIP sensors. Calibration curves obtained for the detection of the pyrene at the MIP sensors in aqueous media reported limits of detection (LOD) of $2.28 \times$ $10^{-7} \mathrm{M}$ for pyrene and limit of quantification (LOQ) of $6.92 \times 10^{-7} \mathrm{M}(n=3)$. The sensitivity of the MIP sensors $(32.53 \mathrm{~A} / \mathrm{M})$ determined from the slopes of the calibration curves reported twice the value measured for NIP sensors (14.48 A/M). The selectivity of the MIP sensors was further evaluated in the presence of a second PAH with the same number of rings as the imprinted PAH, i.e., chrysene, to evaluate the selectivity of the MIP sensor towards shape and size of the analyte.
\end{abstract}

Keywords Electrochemical sensors $\cdot$ Molecularly imprinted polymers $\cdot$ Polyaromatic hydrocarbons

\section{Introduction}

Polycyclic aromatic hydrocarbons (PAH) are a class of complex organic chemicals, consisting of a fused ring structure with at least 2 benzene ring [1,2]. In general, these compounds are formed as a result of pyrolytic processes, such as the incomplete combustion of organic materials during industrial and other human activities, under conditions where organic substances are exposed to high temperatures under low oxygen or no oxygen conditions [3]. During pyrolysis, a wide range of PAH is formed and the US Environmental Protection Agency (EPA) has put together a list of 16 PAHs as priority pollutants $[4,5]$. This list was created to classify PAH according to the toxicity, carcinogenicity, and mutagenicity in both humans and animals.

Priscilla G. L. Baker

pbaker@uwc.ac.za

1 SensorLab research group, Chemistry Department, University of the Western Cape, PB X17, Bellville 7535, South Africa
PAHs may be synthesized from saturated hydrocarbons under oxygen-deficient conditions. Moreover, two other ways to account for the formation of PAHs are pyrosynthesis and pyrolysis (Scheme 1). Low hydrocarbons form PAHs by means of pyrosynthesis. When temperatures exceed $500{ }^{\circ} \mathrm{C}$, the carbon-hydrogen and carbon-carbon bonds are broken to form free radicals. These radicals combine to acetylene which further condenses into aromatic ring structures, which are resistant to thermal degradation. The formation of the benzene ring (monomer) is the common step preceding the synthesis of polyaromatic hydrocarbons $[6,7]$.

Pyrene is a polycyclic aromatic hydrocarbon, consisting of four fused benzene rings, which is produced during incomplete combustion of organic compounds [8]. The EPA advocates a reference dose $(\mathrm{RfD})$ of $0.03 \mathrm{mg} / \mathrm{kg} /$ day as an estimate of a daily oral exposure to the human population that is likely to be without an appreciable risk of deleterious effects during a lifetime [9]. The structure of pyrene is shown below along with other four membered ring PAHs to illustrate the seemingly small differences in structure that give rise to very distinct chemical interactions (Fig. 1). The commonly used 
Scheme 1 Pyrosynthesis reaction of PAHs with ethyne as a starting material

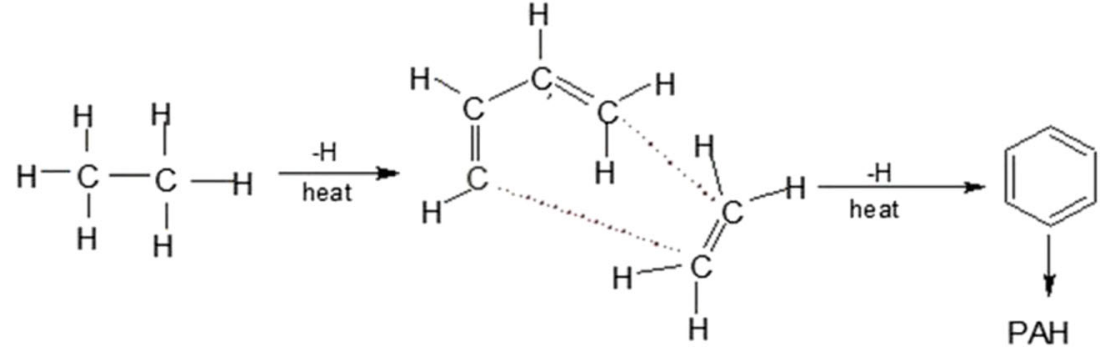

analytical methods for the detection of PAHs from industrial samples are high performance liquid chromatography (HPLC) coupled to fluorescence detection, membrane filtration, ozonation, and reverse osmosis. Analysis of PAHs from the petrochemical industry is typically performed by HPLC method as well as sono-degradation in the presence of oxygen and hydrogen peroxide [10].

In this study, polypyrrole (Ppy) was chosen as a suitable polymer for imprinting of pyrene, because it can be electrochemically synthesized in situ with good control over film thickness and with good semiconductor properties. Ppy has been widely used as an electrochemical transducer due to its low cost, semi-conductive behavior, efficient electrochemical properties, and stability. Ozcan et al. have used Ppy in the determination of paracetamol at electropolymerized molecularly imprinted polymer (MIP)-modified pencil graphite electrodes [11]. The polymer was prepared by electrosynthesizing pyrrole through galvanostatic deposition or cyclic voltammetry to obtain Ppy thin films. These methods provide a simple and rapid way of controlling the thickness of the polymer film growth since polymer thickness needs serious consideration in MIP [12]. Schweiger et al. in their work of sensing clofibric acid using MIP based on Ppy explained that these conducting polymers possess metal-like behavior due to the conjugated double bonds. [13]. The structure and morphology of polypyrrole may be controlled by a wide range of electrosynthesis conditions such as scan rate, number of cycles, but the counter ions play the most important role, since the electrochemical method produces the polymer in its oxidized and doped state (Scheme 2).

Ppy films have an overall hydrophilic character which makes it ideal for detection of organic compounds through surface hydrogen bonding [14]. Oxidative polymerization of pyrrole to polypyrrole proceeds via a one electron oxidation of pyrrole to a radical cation, which subsequently couples with another radical cation to form the 2,2'-bipyrrole. The resulting polymer is produced in the oxidized state, incorporating the counter ions from electrolyte solution $\left(\mathrm{Cl}^{-}\right)$to stabilize the positively charged polymer chain. This process is then repeated to form longer chains, and during the chain formation, a molecular imprint may be generated when the target molecule is present in the polymerization solution.

Molecular imprinting technology (MIT) offers a process of preparing materials with cavities that are able to recognize and attract a certain molecule of interest in terms of shape, size, and chemical functionality [15]. The molecule of interest (analyte) needs to be incorporated during the synthesis of the material, where it serves as a template. When synthesis reaches completion, the molecule is extracted, leaving behind its 3D physical and chemical "imprint." These cavities are capable of selectively rebinding the template molecule even in the presence of close structural analogs.

In this paper, we report on highly sensitive and selective detection of pyrene from aqueous solution, at molecularly imprinted polypyrrole electrodes, prepared by electrochemical synthesis methods.

\section{Experimental}

\section{Materials}

\section{Chemicals and reagents}

The pyrrole monomer (98\%), pyrene (98\%), chrysene (98\%), methanol (99\%), and hydrochloric acid (99\%) were all purchased from Sigma Aldrich. The alumina polishing beads were purchased from Buelher Chemical company. Ultrapure water (Millipore) was used for all preparations.
Fig. 1 Pyrene and its analogous molecules<smiles>c1cc2ccc3cccc4ccc(c1)c2c34</smiles><smiles>c1ccc2cc3c(ccc4ccccc43)cc2c1</smiles>

Pyrene $\left(\mathrm{C}_{16} \mathrm{H}_{10}\right)$
Benzo[a] anthracene $\left(\mathrm{C}_{18} \mathrm{H}_{12}\right)$<smiles>c1ccc2c(c1)ccc1c3ccccc3ccc21</smiles>

Pyrene $\left(\mathrm{C}_{18} \mathrm{H}_{12}\right)$ 
Scheme 2 Electropolymerization of polypyrrole n

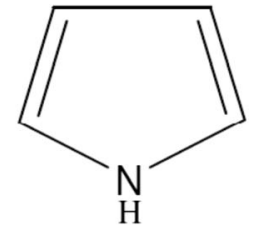

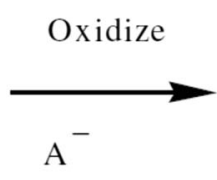

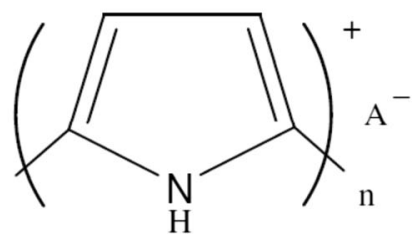

Template preparation

Solutions of $0.0005 \mathrm{M}$ of pyrene and chrysene were prepared by weighing the appropriate mass of the $\mathrm{PAH}$, dissolving it in the required volume of methanol and subsequently sonicating the standard solution, until fully dissolved. The standard solutions were kept in vials in a laboratory cupboard. The UV/vis absorbance was measured over time to verify the integrity of the standard solutions (data not shown).

\section{Preparation of polymer and imprinted electrodes}

A classical three electrode electrochemical cell was used for the electropolymerization of pyrrole, at commercial glassy carbon electrode (GCE) as the working electrode, $\mathrm{Ag} / \mathrm{AgCl}$ $(3 \mathrm{M} \mathrm{NaCl})$ as the reference electrode and a $50 \mathrm{~mm}$ Pt-wire $(d$ $=1 \mathrm{~mm}$ ) as counter electrode. The working electrode was cleaned prior to each experiment, by polishing the electrodes with a micropolish slurry of $1.0 \mu \mathrm{m}, 0.3 \mu \mathrm{m}$, and $0.005 \mu \mathrm{m}$ particle size, respectively. The electrode was then rinsed with deionized water and ultra-sonicated in ethanol and water, consecutively. The pyrrole monomer was freshly distilled before use, in order to ensure purity of the monomer by removing any short chains from spontaneous polymerization due to extended shelf life. The molecularly imprinted polymer electrodes were prepared by introducing the pyrrole monomer and the template molecule (PAH) $0.0005 \mathrm{M}$ standard solution, into 3 $\mathrm{mL}$ of $0.1 \mathrm{M} \mathrm{HCl}$ electrolyte solution in equal molar ratios, to produce a MIP thin film at the GCE interface [16]. The pyrrole monomer is water soluble and through the number of cycles, the polymer films' thickness could be controlled. Smaller counter ions such as $\mathrm{Cl}^{-}$are preferentially included in the growing polymer film to improve the conductivity through p-doping and film thickness does not affect the electrical conductivity of the film [17].

The potential window used for the synthesis was -0.4 to $0.7 \mathrm{~V}$ (vs $\mathrm{Ag} / \mathrm{AgCl}$ ), with 5 cycles of polymerization at 50 $\mathrm{mV} / \mathrm{s}$, under ambient conditions using a Palmsense Trace 4.4 and its operating software. After the electropolymerization in the presence of the template molecule, the MIP thus produced was washed with methanol to remove the template and leave only the imprint behind. The non-imprinted polymer (NIP) electrodes were prepared in the same way as the MIP electrodes, but starting with pyrrole monomer only.

\section{Electrochemistry of PAH}

Cyclic voltammetry in a classical three electrode electrochemical cell was used with the PAH-imprinted polypyrrole film (MIP) at the glassy carbon electrode, connected as the working electrode, $\mathrm{Ag} / \mathrm{AgCl}(3 \mathrm{M} \mathrm{NaCl})$ as a reference and Pt-wire as the counter electrode. Standard additions of the required PAH standard solution $(0.005 \mathrm{M})$ were added to the electrochemical cell and stirred for $60 \mathrm{~s}$. Molecular recognition of PAHs was performed using cyclic voltammetry in order to evaluate the analytical response of the PAH-imprinted polypyrrole sensors. Electrochemistry of the NIP electrodes was performed in a similar electrochemical cell with the NIP, connected as the working electrode.

\section{Interference studies}

The selectivity and sensitivity of the MIP electrode was evaluated in the presence of chrysene, which is a structural analog of pyrene. These two PAH molecules are similar to each other in terms of the number of rings, but the position of ring fusion differs.

\section{Results and discussion}

\section{Material characterizations}

\section{Atomic force microscopy}

Atomic force microscopy (AFM) measurement was performed on a MIP deposited directly onto a glassy carbon electrode surface, using a Nanosurf easyScan 2 (Wirsam) for morphology imaging in non-contact mode. AFM images were taken for the bare electrode, polymer with pyrene before washing and pyrene-imprinted polypyrrole after washing (Fig. 2).

The AFM of the bare electrode was smooth and regularly patterned, whereas the MIP before washing clearly showed the clustering due to the incorporation of the pyrene. After washing, the topography changed to a smooth surface with dark spots indicated by the arrows in Fig. 2c. These dark spots created after pyrene removal may be associated with the imprints of pyrene. Li et al. [18] reported similar AFM images for graphene oxide molecularly imprinted sensor for the detection of endocrine disrupting chemicals. Their study indicated that the topography of the non-imprinted graphene oxide 

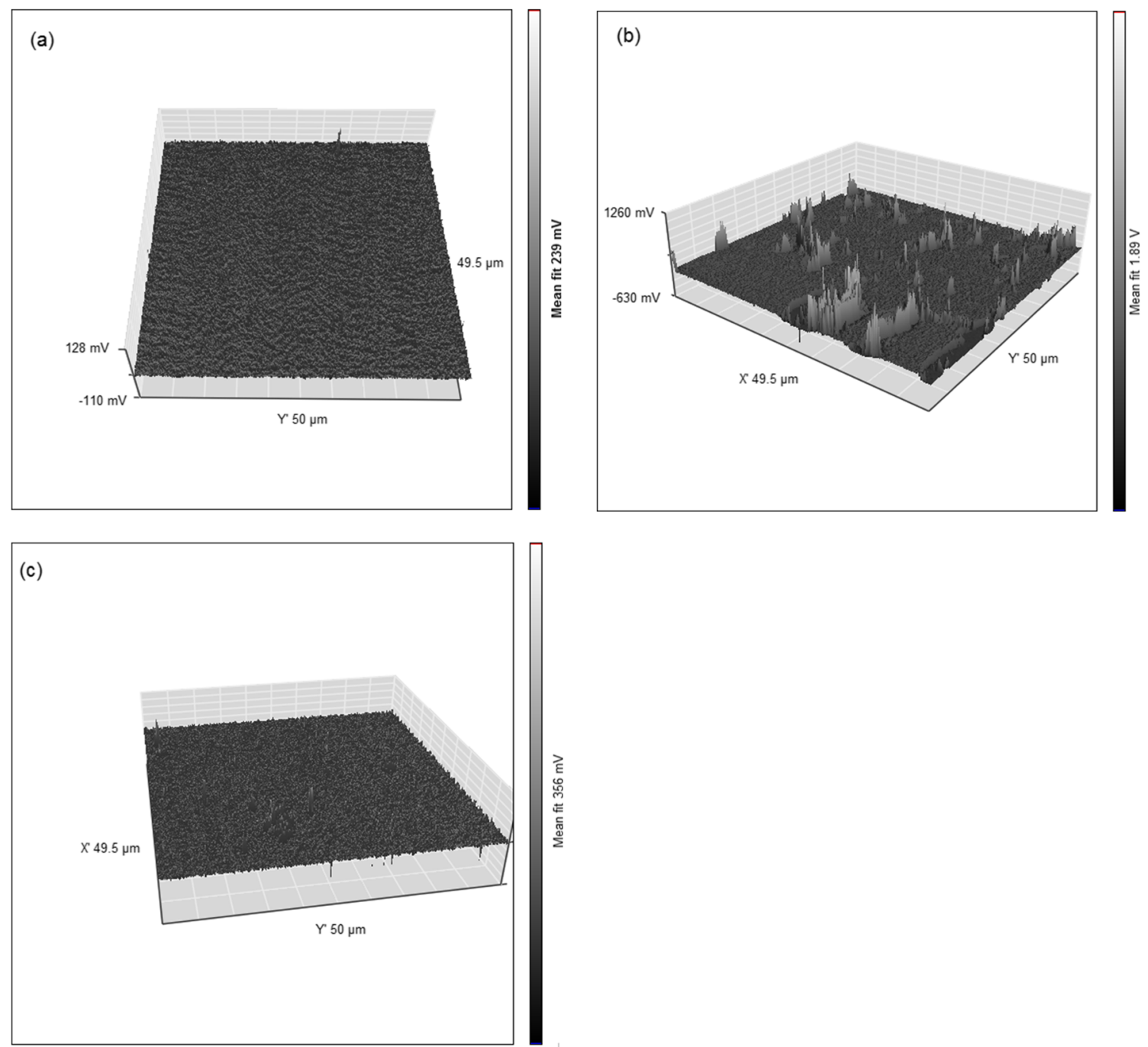

Fig. 2 AFM images of (a) GCE bare electrode, (b) MIP electrode before washing, (c) MIP electrode after washing

was smooth and regular, whereas the topography after imprinting was irregular. The irregular topography was associated with the template cavities imposed on the graphene oxide.

\section{Scanning electron microscopy}

Scanning electron microscopy (SEM) was used to study the surface morphology and the particle size of the NIP and MIP sensors and measurements were performed using a Zeiss Auriga, high-resolution (fegsem) field emission gun scanning electron microscope instrument equipped with secondary electron capture detector, for imaging. The samples were not coated for viewing, and SEM images before and after the removal of pyrene are shown (Fig. 3).

Prior to the removal of pyrene, the particle size was observed to be small and regular at both $200 \mathrm{~nm}$ (a) and $100 \mathrm{~nm}$ resolution (c) compared to after washing the imprinted polymer, when the particle size changed to thick and irregular (b and d). The MIP appeared rough and irregular with more spaces between polymer particles and those properties may be attributed to the formation of the recognizing cavities. Gonzalen et al. [19] have reported similar SEM results for molecularly imprinted polymers, where the NIP electrode showed narrow and clustered particles whereas the MIP electrode showed much thicker particle size and there were spaces created in between the particles. Additionally, the spaces created in the electrode surface were irregular and were attributed to the cavities. Furthermore, charging effects may also be helpful in identifying topography changes. When the incident beam interacts with a sample, part of the incident electrons are emitted back, but a larger fraction is captured by the specimen. This charge flows to ground if the specimen is a uniform conductor. However, if the ground path is broken, even a conducting specimen quickly accumulates charge and its surface potential rises, producing white and dark spots on topography images, respectively. The incorporation of the pyrene template is seen as a darkening of the overall SEM image, indicating a change in charging effects as a result of molecular imprinting [20]. 
Fig. 3 SEM images of (a) NIP and (b) MIP sensors at $200 \mathrm{~nm}$ resolution and (c) NIP and (d) MIP at $100 \mathrm{~nm}$ resolution
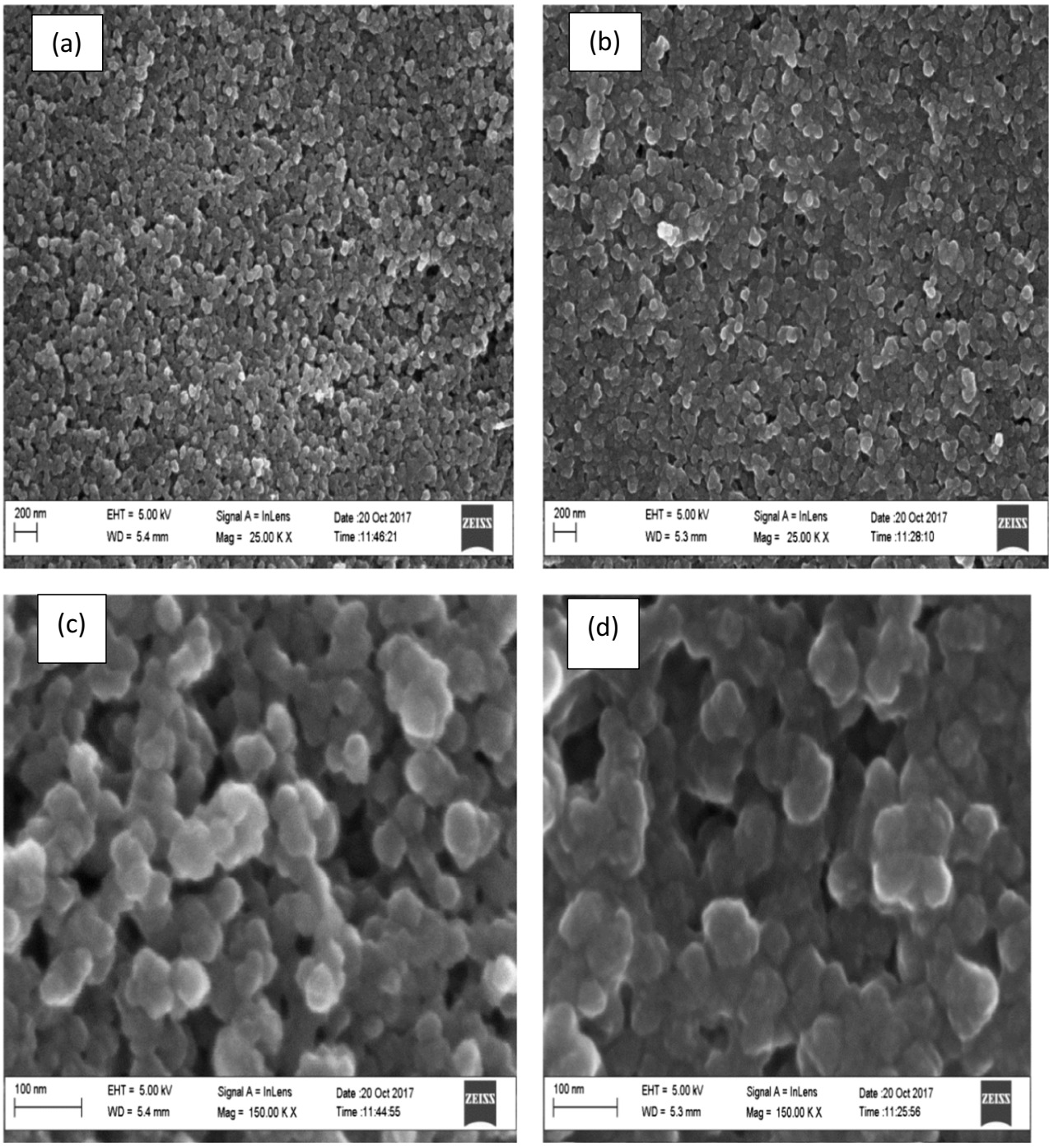

\section{Preparation and characterization of NIP and MIP electrodes}

The electropolymerization of the pyrrole monomer was achieved after 5 cycles at $50 \mathrm{mV} / \mathrm{s}$ between the 0.4 and $0.7 \mathrm{~V} \mathrm{vs} \mathrm{Ag} / \mathrm{AgCl}$ in $0.1 \mathrm{M} \mathrm{HCl}$ at a polished glassy carbon electrode. The film growth was observed as an increase in current with each cycle. The number of cycles were optimized at 5 cycles in order to produce a sufficiently thin layer and imprintable polymer framework for the subsequent modification with the template. The polymer layer built up on the electrode surface was proportional to the increasing current response, indicating an in situ deposited thin film [21].

Characterization of the as-deposited polypyrrole thin film indicated quasi-reversible charge transfer behavior with an oxidation peak (a) and one reduction peak (a') separated by more than $600 \mathrm{mV}$. (Fig. 4). The formal potential $\left(\mathrm{E}^{\circ}\right)$ may be obtained from the average values of the peak potentials, i.e., $E^{\circ}=(E p a+E p c) / 2=675 \mathrm{mV}$ vs $\mathrm{Ag} / \mathrm{AgCl}$. Polypyrrole is known as a conducting polymer in which conduction involves charge transport along the polymer chains, as well as hopping of charge carriers, i.e., holes, polarons, and bipolarons. Polarons and bipolarons are the dominant charge carriers in these polymeric conductors, reducing the band gap of the normally insulating polypyrrole. Counter ions such as $\mathrm{Cl}^{-}$ stabilize the charge on the polymer improving the electronic (as opposed to ionic) conduction in polypyrrole [22]. The polymer formed at the electrode interface was subsequently interrogated at different scan rates ranging from 5 to $50 \mathrm{mV} / \mathrm{s}$ (Fig. 4). The diffusion coefficient was evaluated according to Randles Sevçik equation for an irreversible system. [23, 24]. The De was evaluated using the geometric surface area of the electrode $\left(0.071 \mathrm{~cm}^{2}\right)$ in all calculations. In this way, a relative appreciation of the effect of surface modification could be evaluated.

Reported values for De of polypyrrole thin films range from $10^{-14}$ to $10^{-6} \mathrm{~cm}^{2} / \mathrm{s}$, depending on the counterion used and other synthesis factors [25]. The De for the polypyrrole 
Fig. 4 Characterization of a polypyrrole thin film in $0.1 \mathrm{M}$ $\mathrm{HCl}$ at scan rates ranging from 5 to $50 \mathrm{mV} / \mathrm{s}$, vs $\mathrm{Ag} / \mathrm{AgCl}$

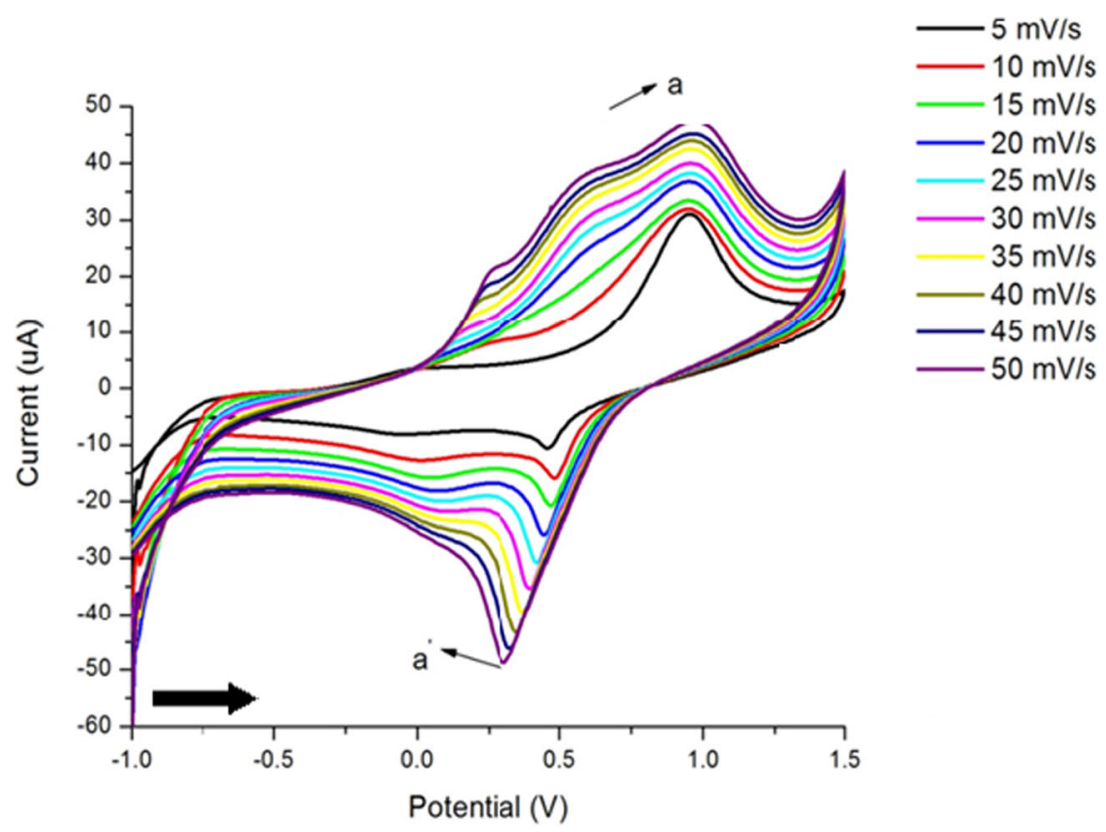

thin film prepared in this work was calculated to be $3.25 \times$ $10^{-12} \mathrm{~cm}^{2} \mathrm{~s}^{-1}$ and in good agreement with recently reported values for polypyrrole synthesized from $\mathrm{HCl}[26,27]$. On the forward scan from $-1000 \mathrm{mV}$ to $1500 \mathrm{mV}$, the dominant oxidation peak at $1000 \mathrm{mV}$ (vs $\mathrm{Ag} / \mathrm{AgCl}$ ) was attributed to the neutral species Ppy being oxidized to Ppy+. On the reverse scan, a reduction peak at $300 \mathrm{mV}$ was attributed to the cationic species, Ppy+ being reduced back to Ppy. The MIP electrode was prepared by electropolymerization of the pyrrole monomer in the presence of $0.0005 \mathrm{M}$ pyrene as the template molecule. The imprinted polymer electrode, after washing with methanol to remove the template molecule, was characterized in the same way as the NIP electrode.

a

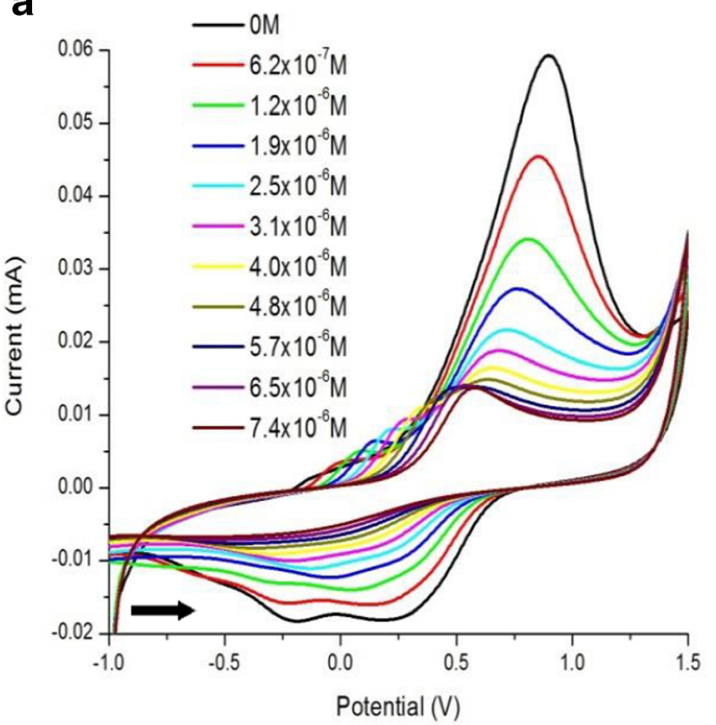

\section{Analytical response of pyrene}

\section{Pyrene-imprinted polypyrrole}

Cyclic voltammetry was used in the analytical evaluation of pyrene redox behavior at the NIP-modified GCE as well as the MIP-modified GCE (Fig. 5). The decrease in oxidative peak current at $1000 \mathrm{mV}$ vs $\mathrm{Ag} / \mathrm{AgCl}$ was in direct response to the consecutive addition of $4 \mu \mathrm{L}$ of $0.0005 \mathrm{M}$ pyrene standard solution to $3.00 \mathrm{~mL}$ cell volume, resulting in a final analytical concentration range of $6.2 \times 10^{-7}$ to $7.4 \times 10^{-6} \mathrm{M}$.

PAH typically have high oxidation potentials and the ring opening expected from full oxidation in practice may be

b

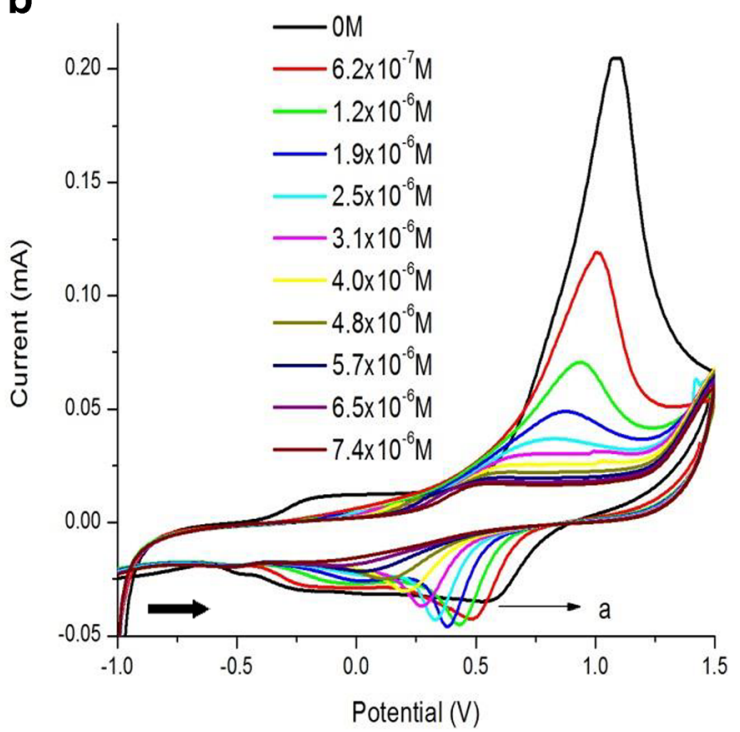

Fig. 5 Cyclic voltammetry of the concentration-dependent response of the (a) NIP electrode and the (b) MIP electrode towards pyrene detection in $0.1 \mathrm{M}$ $\mathrm{HCl}$ at $50 \mathrm{mV} / \mathrm{s}$ 
1<smiles>CC(C)C</smiles><smiles>[CH]1C=CC2C=Cc3cccc4ccc1c2c34</smiles><smiles>C[CH+]C</smiles>

o<smiles>OC1C=CC2C=Cc3cccc4ccc1c2c34</smiles><smiles>O=C1C=CC2=C3C4=C(C=C4)C=CC1C=CC3C=C2</smiles><smiles>Oc1ccc2ccc3c(O)ccc4ccc1c2c43</smiles>
$+4$

2<smiles>Oc1ccc2ccc3cccc4ccc1c2c34</smiles>

3

Scheme 3 Mechanism for the electrochemical oxidation of pyrene

achieved using harsh chemical reagents, such as Fenton's reagent. Surface bound electrochemical oxidation of pyrene has been reported to yield highly redox-active surface confined quinone species at $1 \mathrm{~V}$ vs $\mathrm{Ag} / \mathrm{AgCl}$ in $\mathrm{pH} 7, \mathrm{PBS}$ at high surface area carbon electrodes [28]. The electrochemically driven electronic rearrangement within the pyrene ring structure during the controlled potential cycling resulted in a sharp analytical peak response at $1000 \mathrm{mV}(\mathrm{vs} \mathrm{Ag} / \mathrm{AgCl})$ indicating a complimentary effect between the template cavity in the
MIP and the analyte in solution (Scheme 3). The NH groups of the pyrrole moieties act as the $\pi-\pi$ interaction donors while the aromatic ring of pyrene functions as an $\pi-\pi$ acceptor. These interactions, together with the size and shape of the pyrene binding sites, lead to a highly sensitive and specific analytical signal for pyrene [29].

The oxidation current at $1000 \mathrm{mV}(\mathrm{vs} \mathrm{Ag} / \mathrm{AgCl})$ was used as the analytical peak for the construction of the respective calibration curves and showed a decreasing trend with each
Fig. 6 Calibration curves for pyrene detection at MIP sensor (black dot) and NIP sensor (black diamond) at a scan rate of $50 \mathrm{mV} /$ $\mathrm{s}$ in a $0.1 \mathrm{M} \mathrm{HCl}(N=3)$

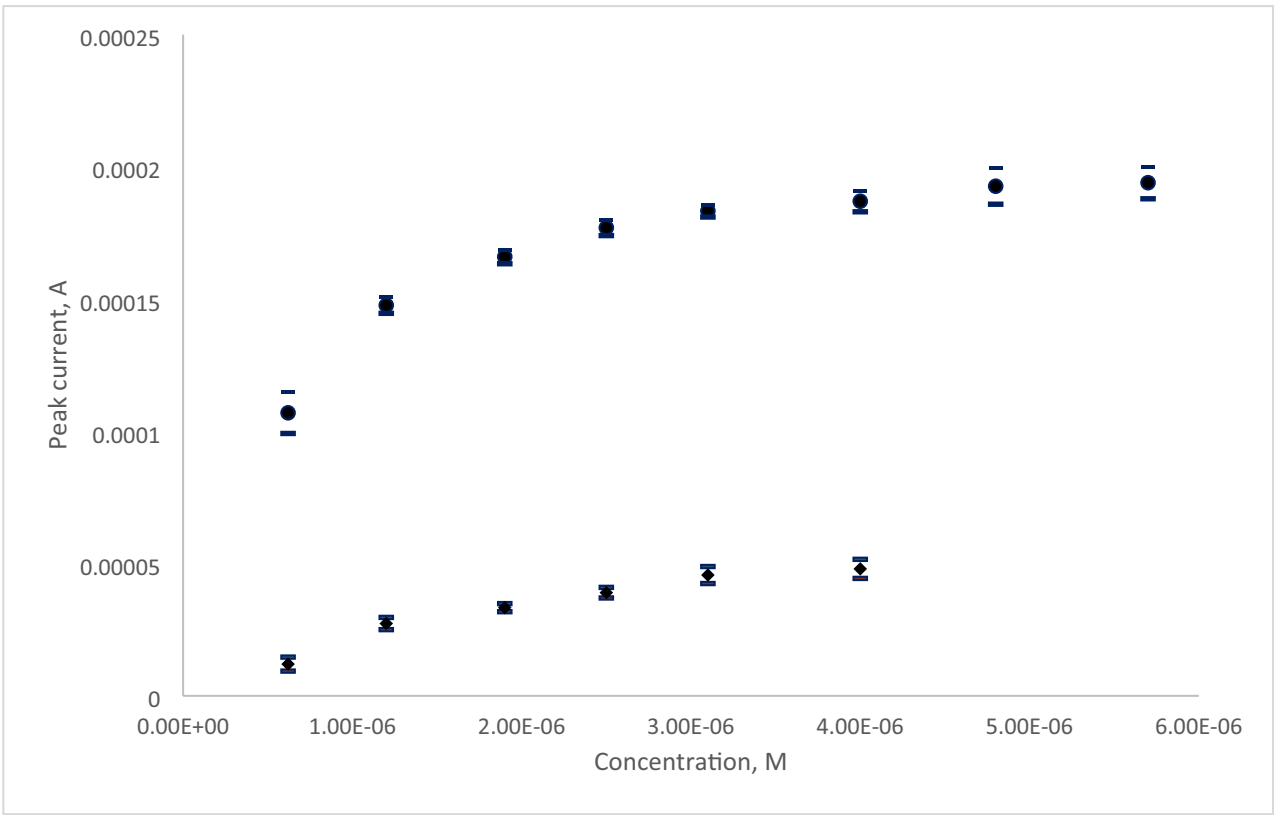




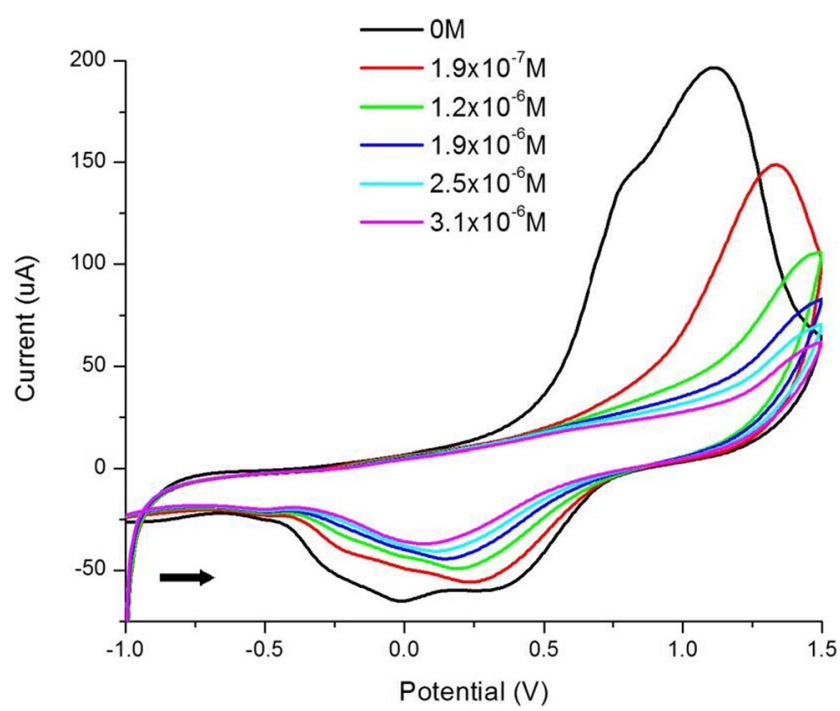

Fig. 7 Cyclic voltammogram showing response of the pyrene-imprinted sensor (MIP) towards increasing concentration of chrysene, at a scan rate of $2 \mathrm{mV} / \mathrm{s}$ in $0.1 \mathrm{M} \mathrm{HCl}$

addition of pyrene. The decreasing trend in current was ascribed to the insulating effect as the nonconductive pyrene fits into the imprint of the polymer (MIP) or adsorbs onto the polymer (NIP). However, higher currents and sharper peaks were observed at the MIP transducer, indicating improved sensitivity and selectivity of the MIP transducer, due to the shape and size recognition features. Both the MIP and NIP sensors reported decreasing current with each addition of analyte and the calibration curves were normalized with respect to the peak current before adding analyte $(0 \mathrm{M})$. The same concentration range was used in the evaluation of NIP and MIP sensors, for direct comparison of sensor performance and the experiments were conducted in triplicate (Fig. 6).

The MIP sensor reported a higher current response $\left(10^{2}\right.$ $\mu \mathrm{A}$ ) for each concentration measured compared to that of the NIP sensor $\left(10^{1} \mu \mathrm{A}\right)$ which indicates the improved sensitivity towards pyrene due to the templating effect. The shape of both calibration curves reached a plateau when the surface concentration of pyrene reached a maximum. This means that the NIP and MIP sensors may only be used for once off analysis, with the advantage of real-time reporting of pyrene detection. Evaluation of the linear range of the calibration curve at lower concentrations, reported a limit of detection of 4.23 $\mathrm{x} 10^{-7} \mathrm{M}(\mathrm{S} / \mathrm{N}=3)$ for NIP sensors compared to $2.28 \times 10^{-7} \mathrm{M}$ for MIP sensors, within the concentration range evaluated. The MIP sensor reported a superior sensitivity of $32.53 \mathrm{~A} / \mathrm{M}$ compared to that of the NIP sensor, i.e., $14.48 \mathrm{~A} / \mathrm{M}$, as calculated from the slope of the linear range of calibration curve.

\section{Interference studies with chrysene}

The selectivity of the MIP electrode in this work was evaluated in the presence of one other analogous PAH to investigate the structural selectivity of the MIP sensor. In the first interference experiment, chrysene was introduced as the analyte in a three electrode electrochemical cell, where the working electrode was the pyrene-imprinted polymer electrode (as before). The reference and counter electrodes were the same as before and the only difference therefore was the analyte prepared as a $0.0005-\mathrm{M}$ chrysene solution. It was expected that the pyrene-imprinted sensor should have a strong affinity to pyrene, even in the presence of the structural analog chrysene, due to the shape and size recognition effect of the imprinted template. In addition to the geometric complementarity of the imprinted cavity, sensitivity for the template is enhanced due to $\pi$-donor and hydrogen bonding interactions with pyrene [29]. The final analytical concentration range for chrysene detection was as before, $6.2 \times 10^{-7} \mathrm{M}$ to $7.4 \times 10^{-6} \mathrm{M}$ (Fig. 7).

The analytical response of a pyrene-imprinted sensor towards the detection of chrysene was not observed at $50 \mathrm{mV} /$ $\mathrm{s}$ and upon further investigation, some concentrationdependent behavior was only observed at very slow scan rate.
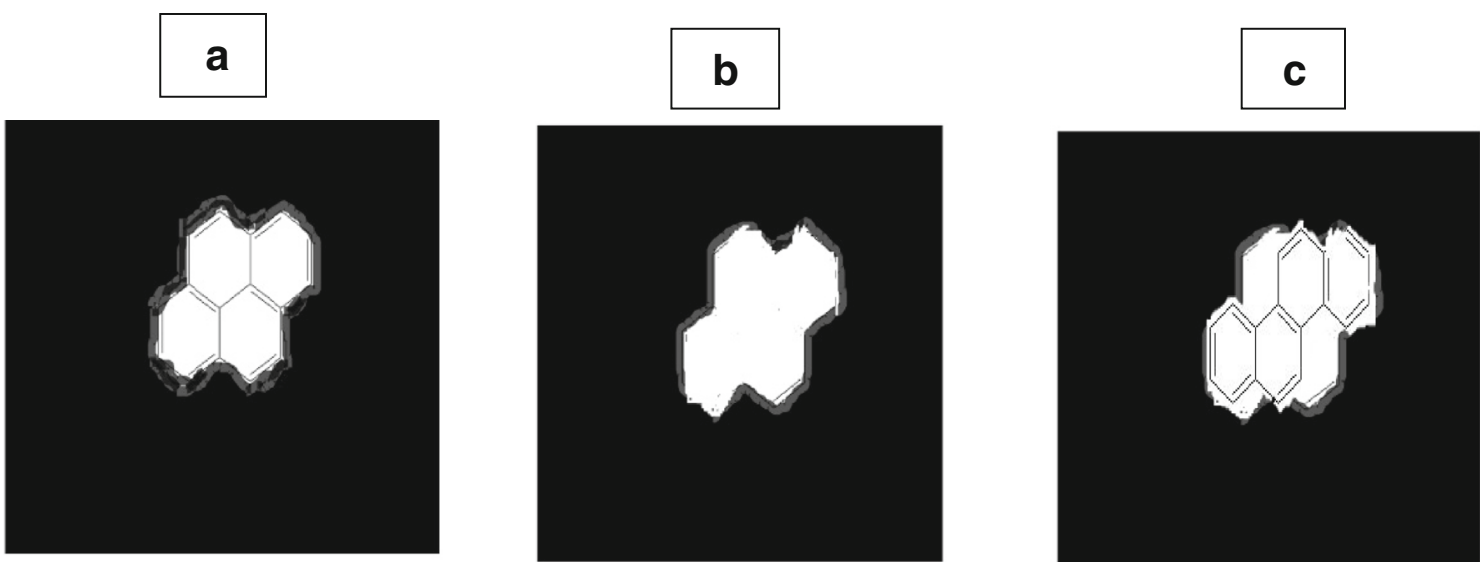

Fig. 8 Diagram of (a) pyrene-imprinted polypyrrole, (b) pyrene-imprinted polypyrrole sensor after template removal, and (c) proposed poor fit of chrysene at the pyrene-imprinted polypyrrole sensor 
Fig. 9 Calibration curve for chrysene at pyrene MIP sensor $(N$ $=3$ )

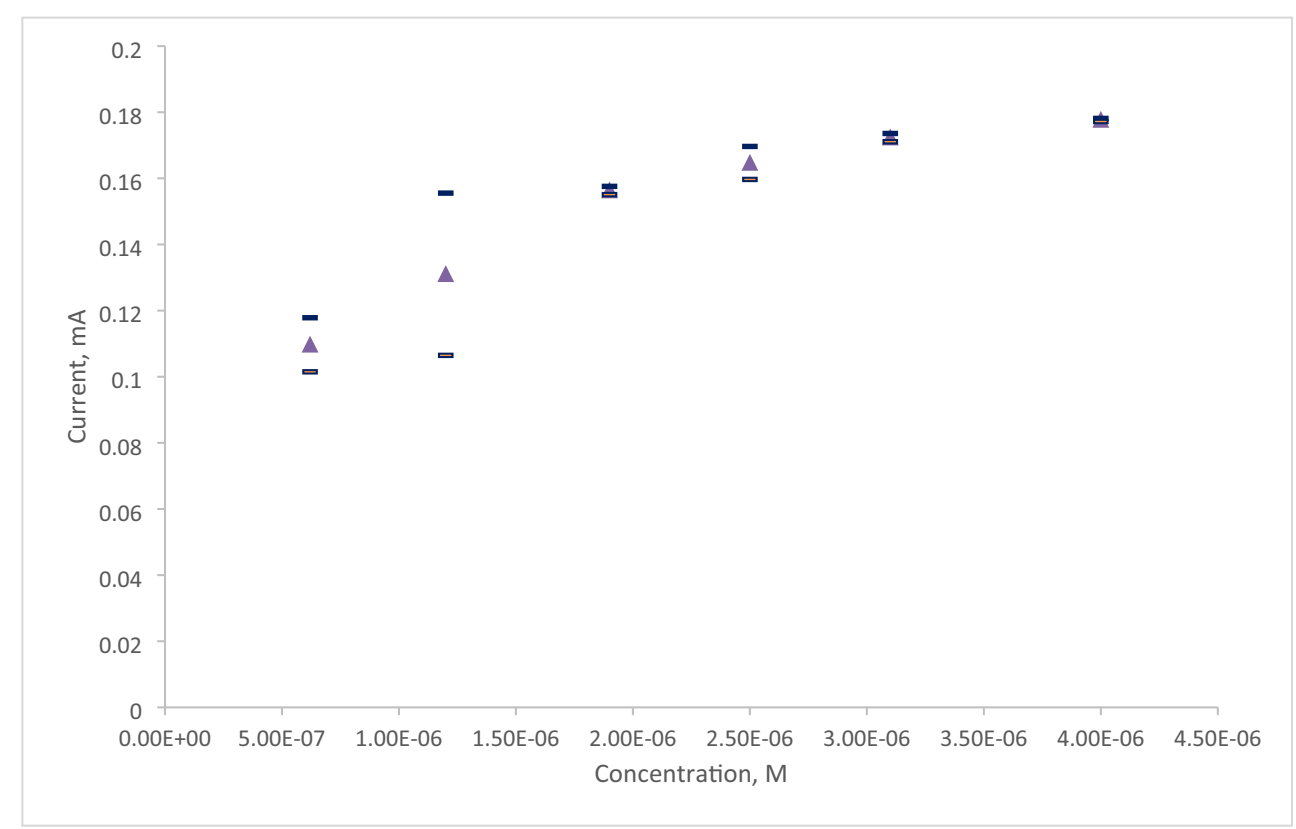

The same adsorptive behavior as for pyrene at MIP and MIP was indicated by the decreasing peak current when the scan rate was adjusted to $2 \mathrm{mV} / \mathrm{s}$. However, the oxidation peak potential was observed at $1.25 \mathrm{~V}$ vs $\mathrm{Ag} / \mathrm{AgCl}$ and shifted to higher positive potentials with each consecutive addition of chrysene. The slower scan rate required for analytical response, the changing peak current shape and intensity, as well as the shifting peak potential were the key factors that indicated a mismatch between the pyrene imprint (transducer) and the geometric analog chrysene (in solution), resulting in an irregular quantitative response under the standardized analytical conditions and hence a calibration curve for chrysene detection at the pyrene-imprinted polymer sensor interface was not possible (Fig. 8). Pyrene is a flat 4 membered ring structure with a different line of symmetry to chrysene, another flat 4 membered ring, i.e., the two analyte molecules are not congruent. Hence, only the molecule with the correct shape and size will match with the polymer imprint, resulting in the desired sharp analytical response.

The analytical oxidation peak was used to construct a calibration, using the same concentration range as before to enable direct comparison (Fig. 9).

In a second interference experiment, an equimolar mixture of $0.0005 \mathrm{M}$ pyrene and $0.0005 \mathrm{M}$ chrysene was prepared, to evaluate the sensitivity of the MIP to its target, from a mixture containing both structural analogs. Cyclic voltammetry was employed, using the original parameters used for pyrene detection, for comparison with earlier experiments of pyrene detection at NIP and MIP.

The cyclic voltammogram (Fig. 10) shows that the oxidation peak observed for the pyrene-imprinted sensor before was not altered by the presence of the chrysene, and the signaling peaks could still be used for the construction of calibrations curve as we have done before (Fig. 11).

The calibration curves show no major difference in sensitivity of the pyrene-imprinted polymer electrode for pyrene detection as a single analyte (32.53 A/M) compared to pyrene detection from a mixture of pyrene and chrysene (31.01 A/M). This strongly supports the conclusion that imprinted polymers are able to discriminate the target even when presented with structural analogs and that molecularly imprinted polymers are highly sensitive to the target molecule. Analytical protocols for detection and quantification of polyaromatic hydrocarbons and their derivatives still strongly rely on spectroscopic measurement [30]. MIP technology using

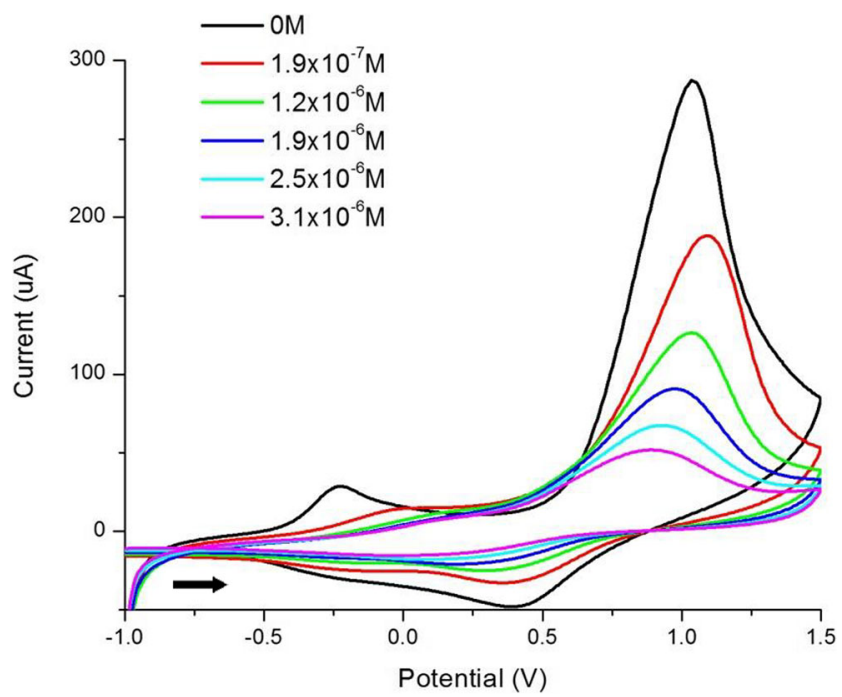

Fig. 10 Analytical response of the MIP sensor towards pyrene from a mixed chrysene and pyrene solution, at $50 \mathrm{mV} / \mathrm{s}$ in $01 \mathrm{HCl}$ 
Fig. 11 Calibration curves for detection of pyrene only (black square) and pyrene in the mixed solution with chrysene (red circle) at the MIP sensor, in $0.1 \mathrm{M} \mathrm{HCl}$ at scan rate of $50 \mathrm{mV} / \mathrm{s}$

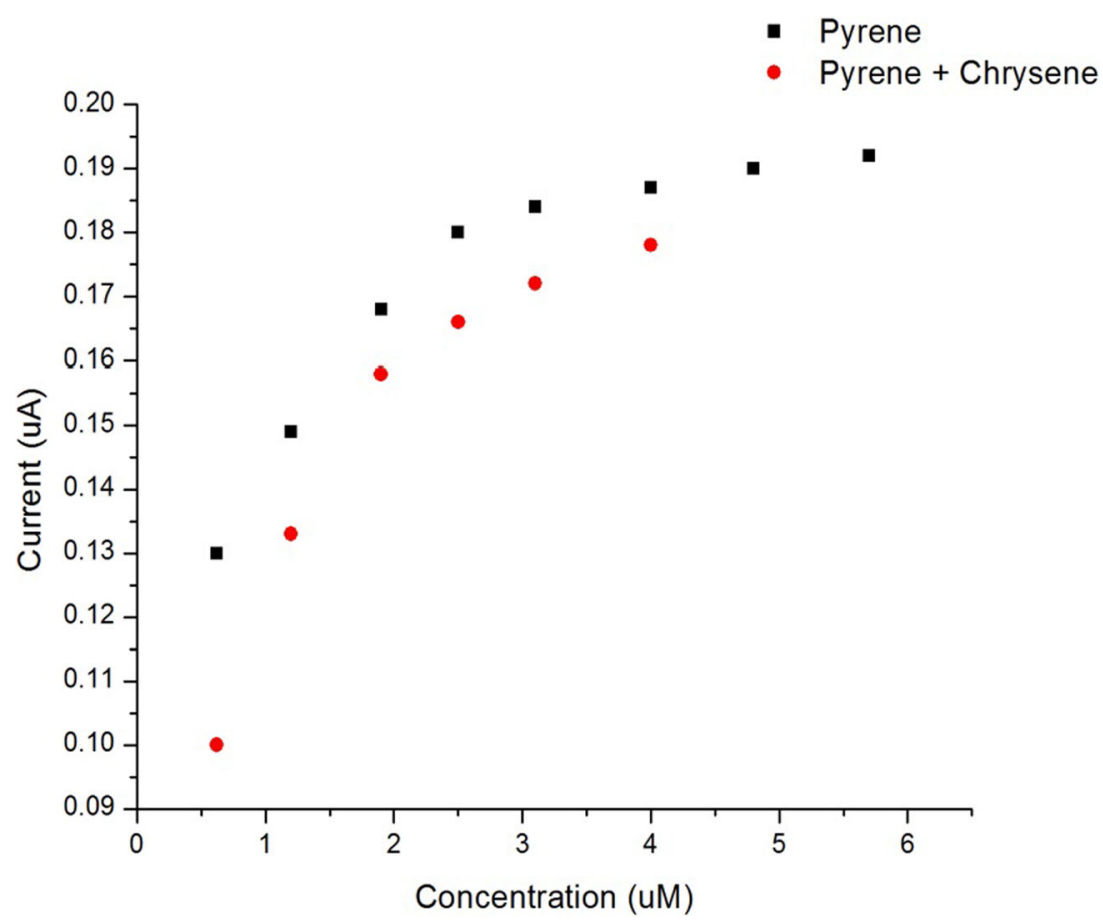

electrochemically conductive polymers is widely recognized as an efficient method of chemical sensing [31]. Literature reports for pyrene detection at molecularly imprinted polypyrrole sensors are limited; in fact, the MIP sensors reported for pyrene detection are typically based on polythiophene. We have demonstrated here that molecular imprinting of polypyrrole shows great promise for sensitive and selective reporting of polyaromatic hydrocarbons in aqueous medium.

\section{Conclusions}

Molecular imprinting of polymers rely heavily on the characteristics of the cavity for the sensitive and selective recognition of the $\mathrm{PAH}$ analyte molecule. In this work, we have demonstrated the use of polypyrrole in the design of MIP sensors for the detection of trace levels of pyrene in aqueous media with highly selective affinity binding even in the presence of a structural analog. The nanosensor was able to discriminate between pyrene and chrysene based on structural recognition and was able to detect pyrene with good sensitivity from a single sample pyrene solution as well as from a mixed solution of chrysene and pyrene. The system developed in this work therefore holds great promise for discrimination of individual $\mathrm{PAH}$ species in environmental monitoring, in future work.

Funding The authors wish to acknowledge the National Research Foundation (South Africa) as well as the Water Research Commission (South Africa) for funding of this project.

\section{References}

1. R.J. Krupadam, M.S. Khan, S.R. Wate, Removal of probable human carcinogenic polycyclic aromatic hydrocarbons from contaminated water using molecularly imprinted polymer. Water Research 44(3), 681-688 (2010)

2. K. Srogi, Monitoring of environmental exposure to polycyclic aromatic hydrocarbons: a review. Environmental Chemistry Letters 5(4), 169-195 (2007)

3. R. Crisafully, M. Aparecida, L. Milhome, R.M. Cavalcante, E.R. Silveira, D. De Keukeleire, R.F. Nascimento, Removal of some polycyclic aromatic hydrocarbons from petrochemical wastewater using low-cost adsorbents of natural origin. Bioresources Technology 99(10), 4515-4519 (2008)

4. Y. Ma, S. Harrad, Spatiotemporal analysis and human exposure assessment on polycyclic aromatic hydrocarbons in indoor air, settled house dust, and diet: a review. Environment International 84, 7-16 (2015)

5. A. Aragón, R.M. Toledano, A. Vázquez, J. Villén, J.M. Cortés, Analysis of polycyclic aromatic hydrocarbons in aqueous samples by large volume injection gas chromatography-mass spectrometry using the through oven transfer adsorption desorption interface. Talanta. 139, 1-5 (2015)

6. K. Ravindra, R. van Grieken, Atmospheric polycyclic aromatic hydrocarbons: source attribution, emission factors and regulation. Atmospheric Environment 42(13), 2895-2921 (2008)

7. V.V. Kislov, A.I. Sadovnikov, A.M. Mebel, Formation mechanisms of polycyclic aromatic hydrocarbons beyond the second aromatic ring. The Journal of Physical Chemistry A 117(23), 4794 4816 (2013)

8. A. Penezić, B. Gašparović, D. Stipaničev, A. Nelson, In situ electrochemical method for detecting freely dissolved polycyclic aromatic hydrocarbons in water. Environmental Chemistry 11, 2014, $173-2180$

9. United States Environmental protection agency, 2018 Edition of the Drinking Water Standards and Health Advisories. EPA 822-F-18001 
10. K. Bhupander, V.K. Verma, R. Gaur, S. Kumar, C.S. Sharma, A.B. Akolkar, Validation of HPLC method for determination of priority polycyclic aromatic hydrocarbons (PAHS) in waste water and sediments. Advances in Applied Science Research 5(1), 201-209 (2014)

11. L. Özcan, Y. Șahin, Determination of paracetamol based on electropolymerized-molecularly imprinted polypyrrole modified pencil graphite electrode. Sensors Actuators, B Chem. 127(2), 362-369 (2007)

12. S. Piletsky, A.P.F. Turner, Electrochemical sensors based on molecularly imprinted polymers. Electroanalysis 14(5), 317-323 (2002)

13. B. Schweiger, J. Kim, Y.J. Kim, M. Ulbricht, Electropolymerized molecularly imprinted polypyrrole film for sensing of clofibric acid. Sensors 15(3), 5870-5889 (2015)

14. H.R. Hwang, J.G. Roh, D.D. Lee, J.O. Lim, J.S. Huh, Sensing behavior of the polypyrrole and polyaniline sensor for several volatile organic compounds. Metals and Materials International volume 9(3), 287-291 (2003)

15. P. Spégel, L. Schweitz, S. Nilsson, Molecularly imprinted polymers. Anal. Bioanal. Chem. 372(1), 37-38 (2002)

16. U. Paramo-Garcia, N. Batina, J.G. Ibanez, The effect of $\mathrm{pH}$ on the morphology of electrochemically grown polypyrrole films: an AFM study. Int. J. Electrochem. Sci. 7, 12316-12325 (2012)

17. T. Patois, B. Lakard, S. Monney, X. Roizard, P. Fievet, Characterization of the surface properties of polypyrrole films: influence of electrodeposition parameters. Synthetic Metals 161(2122), 2498-2505 (2011)

18. Y. Li, X. Li, A graphene oxide-based molecularly imprinted polymer platform for detecting endocrine disrupting chemicals. Carbon 48(12), 3427-3433 (2010)

19. G.P. Gonzalez, P. Fern, J.S.D. Alegr, A morphological study of molecularly imprinted polymers using the scanning electron microscope. Analytica Chimica Acta 557(1-2), 179-183 (2006)

20. K.H. Kim, Z. Akase, T. Suzuki, D. Shindo, Characterization of the surface properties of polypyrrole films: influence of electrodeposition parameters. Materials Transactions 51(6), 1080-1083 (2010)

21. T.-P. Huynh, A. Pietrzyk-Le, C. Bikram, K.C. Krzysztof, R. Noworyta, J.W. Sobczak, P.S. Sharma, F. D'Souza, W. Kutner, Electrochemically synthesized molecularly imprinted polymer of thiophene derivatives for flow-injection analysis determination of adenosine-5'-triphosphate (ATP). Biosensors Bioelectronics 41, 634-641 (2013)
22. R. Ansari, Polypyrrole conducting electroactive polymers: synthesis and stability studies. E-Journal of Chemistry 3(4), 186-201 (2006)

23. S. Mokrane, L. Makhloufi, H. Hammache, B. Saidani, Electropolymerization of polypyrrole, modified with germanium, on a passivated titanium electrode in aqueous nitrate solution: new results on catalytic reduction of protons and dissolved oxygen. Journal of Solid State Electrochemistry 5(5), 339-347 (2001)

24. J.B. Sheppard, B. Hambly, B. Pendley, E. Lindner, Voltammetric determination of diffusion coefficients in polymer membranes. Analyst. 142(6), 930-937 (2017)

25. I.J. Suárez, T.F. Otero, M. Márquez, Diffusion coefficients in swelling polypyrrole: ESCR and Cottrell models. J. Phys. Chem. B 109(5), 1723-1729 (2005)

26. E.H. Hess, T. Waryo, O.A. Sadik, E.I. Iwuoha, P.G.L. Baker, Constitution of novel polyamic acid/polypyrrole composite films by in-situ electropolymerization. Electrochimica Acta (128), 4394471 (2014)

27. R. Akinyeye, I. Michira, M. Sekota, A. Al Ahmed, D. Tito, P. Baker, C. Brett, M. Kalaji, E. Iwuoha, Electrochemical synthesis and characterization of 1,2-naphthaquinone-4-sulfonic acid doped polypyrrole. Electroanalysis 19(2-3), 303-309 (2007)

28. B.T.B. Tiu, R.J. Krupadam, R.C. Advincula, Pyrene-imprinted polythiophene sensors for detection of polycyclic aromatic hydrocarbons, sensors and actuators B: chemical sensors actuators B. Chem. 228, 693-701 (2016)

29. P. Barathi, A.S. Kumar, Electrochemical conversion of unreactive pyrene to highly redox active 1,2-quinone derivatives on a carbon nanotube-modified gold electrode surface and its selective hydrogen peroxide sensing. Langmuir 29, 10617-10623 (2013)

30. X. Gong, D. Heeran, Q. Zhao, C. Zheng, D. Yufit, G. Sandford, Synthesis of fluoro and cyanoaryl-containing pyrene derivatives and their optical and electrochemical properties. Asian journal of Organic Chemistry 8(5), 722-730 (2019)

31. P.S. Sharma, A. Pietrzyk-Le, F. D'Souza, W. Kutner, Electrochemically synthesized polymers in molecular imprinting for chemical sensing. Anal Bioanal Chem 402(10), 3177-3204 (2012)

Publisher's Note Springer Nature remains neutral with regard to jurisdictional claims in published maps and institutional affiliations. 